\title{
Soft Set Theory Applied to Hoops
}

\author{
R. A. Borzooei, E. Babaei, Y. B. Jun \\ M. Aaly Kologani and M. Mohseni Takallo
}

\begin{abstract}
In this paper, we introduced the concept of a soft hoop and we investigated some of their properties. Then, we established different types of intersections and unions of the family of soft hoops. We defined two operations $\odot$ and $\rightarrow$ on the set of all soft hoops and we proved that with these operations, it is a hoop and also is a Heyting algebra. Finally we introduced a congruence relation on the set of all soft hoops and we investigated the quotient of it.
\end{abstract}

\section{Introduction}

Hoops are naturally ordered commutative residuated integral monoids, introduced by B. Bosbach in $[11,12]$ then study by J. R. Büchi and T. M. Owens in [13], a paper never published. In the last years, hoops theory was enriched with deep structure theorems(see $[2,4,5,7,8,9,10,15,23])$. Many of these results have a strong impact with fuzzy logic. Particularly, from the structure theorem of finite basic hoops one obtains an elegant short proof of the completeness theorem for propositional basic logic, introduced by Hájek in [17]. The algebraic structures corresponding to Hájek's propositional (fuzzy) basic logic, BL-algebras, are particular cases of hoops. The main example of BL-algebras in interval $[0,1]$ endowed with the structure induced by a t-norm. MV-algebras, product algebras and Gödel algebras are the most known classes of BL-algebras. Recent investigations are concerned with non-commutative generalizations for these structures.

Key Words: Soft set, hoop, soft hoop, Heyting algebra.

2010 Mathematics Subject Classification: 06F35, 03G25, 06D72.

Received: 03.04.2019.

Accepted: 23.07.2019. 
On the other hand, soft set theory was initiated by Molodtsov [21] in 1999 as a new mathematical tool for modelling the uncertainties arising from the parametrization of elements of a universe. He mentioned several directions for the applications of soft sets. In fact, before soft set theory, there have been some mathematical theories such as probability theory, fuzzy set theory, rough set theory, vague set theory, and interval mathematics theory for dealing with uncertainties. However, the superiority of the soft set theory compared with other mathematical tools, is its ability of parametrization. Aktas and Cagman [3] compared soft sets to the related concepts of fuzzy sets and rough sets. They also introduced the notion of soft groups. After them, soft algebraic structures have been studied by many authors [14, 18, 19, 20, 24, 25, 26]. Specially, Y. B. Jun [18] introduced and investigated soft BCK/BCI-algebras. Moreover, by combination of fuzzy set theory with soft set theory, fuzzy soft algebraic structures were born. For example, Hadipour et al. [16] defined the notion of fuzzy soft BF-algebra and investigated the level subset, union and intersection, fuzzy soft image and fuzzy soft inverse image of them. The most soft algebraic structures are defined as follows: for a set of parameters $\mathrm{E}$ and a general algebra $\mathrm{X}$, a pair $(F, E)$ is called a soft general algebra over $\mathrm{X}$ if $\mathrm{F}$ is a map of $E$ into the set of all subsets of the set $X$ such that for each $e \in E, F(e)$ is the empty set or a subalgebra of $X$.

In this paper, the concept of a soft hoop is introduced and some examples are provided. Then, different types of intersections and unions of the family of soft hoops are established. We defined two operations $\odot$ and $\rightarrow$ on the set of all soft hoops on a hoop $H$ and a parameters set $E$ and we proved that with these operations is a hoop and also is a Heyting algebra. Finally we introduced a congruence relation on the set of all soft hoops and we investigated the quotient of it.

\section{Preliminaries}

In this section, we recollect some definitions and results which will be used in the following and we shall not cite them every time they are used.

Molodtsov ([21]) defined the soft set in the following way: Let $U$ be an initial universe set and $E$ be a set of parameters. We denote $\rho(U)$ the power set of $U$ and $A \subseteq E$. A pair $(\lambda, A)$ is called a soft set over $U$, where $\lambda$ is a map given by $\lambda: A \rightarrow \rho(U)$. In other words, a soft set over $U$ is a parametrized family of subsets of the universe $U$. For $\varepsilon \in A, \lambda(\varepsilon)$ may be considered as the set of $\varepsilon$-approximate elements of the soft set $(\lambda, A)$. Clearly, a soft set is not a set. For illustration, Molodtsov considered several examples in [21].

Let $(F, A)$ and $(G, B)$ be two soft sets over $U$. Then,

(i) $(F, A)$ is said to be a soft subset of $(G, B)$, denoted by $(F, A) \subseteq(G, B)$, if 
$A \subseteq B$ and $F(a) \subseteq G(a)$, for all $a \in A$.

(ii) Two soft sets $(F, A)$ and $(G, B)$ are said to be a soft equal, denoted by $(F, A)=(G, B)$, if $(F, A) \subseteq(G, B)$ and $(G, B) \subseteq(F, A)$.

Let $U$ be an initial universe and $E$ be the set of parameters such that $A \subseteq E$. Then,

(i) $(F, A)$ is called a relative null soft set (with respect to the parameter set A), denoted by $\phi_{A}$, if $F(a)=\phi$, for all $a \in A$.

(ii) $(G, A)$ is called a relative whole soft set (with respect to the parameter set A), denoted by $U_{A}$, if $G(a)=U$, for all $a \in A$.

The relative whole soft set with respect to the set of parameters $E$ is called the absolute soft set over $U$ and simply denoted by $U_{E}$. In a similar way, the relative null soft set with respect to $E$ is called the null soft set over $U$ and is denoted by $\emptyset_{E}$. We shall denote by $\emptyset_{E}$ the unique soft set over $U$ with an empty parameter set, which is called the empty soft set over $U$. Note that $\emptyset_{\emptyset}$ and $\emptyset_{E}$ are different soft sets over $U$ and $\emptyset_{\emptyset} \subseteq \emptyset_{A} \subseteq(F, A) \subseteq U_{A} \subseteq U_{E}$, for all soft set $(F, A)$ over $U$ (See $[21,22])$.

The next definition introduces three types of intersections and three types of unions of the family of soft sets over a common universe set. For a family $\left\{\left(F_{i}, A_{i}\right) \mid i \in I\right\}$ of soft sets over $U$, we give some definitions as below:

(a) The extended intersection of the family $\left\{\left(F_{i}, A_{i}\right) \mid i \in I\right\}$ is defined as the soft set $\bigcap_{i \in I}\left(F_{i}, A_{i}\right)=(H, C)$, where $C=\bigcup_{i \in I} A_{i}$ and $H(x)=$ $\bigcap_{i \in I(x)} F_{i}(x) ; I(x)=\left\{i \mid x \in A_{i}\right\}$, for all $x \in C$.

(b) The restricted intersection of the family $\left(F_{i}, A_{i}\right)$ is defined as the soft set $\prod_{i \in I}\left(F_{i}, A_{i}\right)=(H, C)$, where $C=\bigcap_{i \in I} A_{i} \neq \emptyset$ and $H(x)=\bigcap_{i \in I} F_{i}(x)$, for all $x \in C$.

(c) The extended union of the family $\left(F_{i}, A_{i}\right)$ is defined as the soft set $\coprod_{i \in I}\left(F_{i}, A_{i}\right)=(H, C)$, where $C=\bigcup_{i \in I} A_{i}, H(x)=\bigcup_{i \in I(x)} F_{i}(x)$, and $I(x)=\left\{i \mid x \in A_{i}\right\}$, for all $x \in C$.

(d) The restricted union of the family $\left(F_{i}, A_{i}\right)$ is defined as the soft set $\bigcup_{i \in I}\left(F_{i}, A_{i}\right)=(H, C)$, where $C=\bigcap_{i \in I} A_{i} \neq \emptyset$ and $H(x)=\bigcup_{i \in I} F_{i}(x)$, for all $x \in C$.

(e) The $\wedge$-intersection of the family $\left(F_{i}, A_{i}\right)$ is defined as the soft set $\bigwedge_{i \in I}\left(F_{i}, A_{i}\right)=(H, C)$, where $C=\prod_{i \in I} A_{i}$ and $H\left(\left(a_{i}\right)_{i \in I}\right)=\bigcap_{i \in I} F_{i}\left(a_{i}\right)$, for all $\left(a_{i}\right)_{i \in I} \in C$. 
(f) The $\vee$-intersection of the family $\left(F_{i}, A_{i}\right)$ is defined as the soft set $\bigvee_{i \in I}\left(F_{i}, A_{i}\right)=(H, C)$, where $C=\prod_{i \in I} A_{i}$ and $H\left(\left(a_{i}\right)_{i \in I}\right)=\bigcup_{i \in I} F_{i}\left(a_{i}\right)$, for all $\left(a_{i}\right)_{i \in I} \in C$.

A hoop is an algebra $(H, \odot, \rightarrow, 1)$ of type $(2,2,0)$ such that, for all $x, y, z \in H$, $(H P 1) \quad(H, \odot, 1)$ is a commutative monoid,

(HP2) $x \rightarrow x=1$,

$(H P 3) \quad(x \odot y) \rightarrow z=x \rightarrow(y \rightarrow z)$,

$(H P 4) \quad x \odot(x \rightarrow y)=y \odot(y \rightarrow x)$.

On hoop $H$ we define $x \leq y$ if and only if $x \rightarrow y=1$. It is easy to see that $" \leq$ " is a partial order relation on $H$. A hoop $H$ is bounded if there is an element $0 \in A$ such that $0 \leq x$, for all $x \in A$. Let $x^{0}=1, x^{n}=x^{n-1} \odot x$, for any $n \in \mathbb{N}$. If $H$ is a bounded hoop, then we define a negation " ' " on $H$ by, $x^{\prime}=x \rightarrow 0$, for all $x \in H$. If $\left(x^{\prime}\right)^{\prime}=x$, for all $x \in H$, then the bounded hoop $H$ is said to have the double negation property, or (DNP) for short (See [15]).

The following proposition provides some properties of hoop.

Proposition 2.1. $[11,12]$ Let $(H, \odot, \rightarrow, 1)$ be a hoop. Then the following conditions hold, for all $x, y, z \in H$,

(i) $(H, \leq)$ is a $\wedge$-semilattice with $x \wedge y=x \odot(x \rightarrow y)$,

(ii) $x \odot y \leq z$ if and only if $x \leq y \rightarrow z$,

(iii) $x \odot y \leq x, y$ and $x \leq y \rightarrow x$,

(iv) $x \rightarrow 1=1$ and $1 \rightarrow x=x$,

(v) $x \leq y$ implies $x \odot z \leq y \odot z, z \rightarrow x \leq z \rightarrow y$ and $y \rightarrow z \leq x \rightarrow z$.

Proposition 2.2. [15] Let $H$ be a bounded hoop. Then the following conditions hold, for all $x, y \in H$,

(i) $x \leq x^{\prime \prime}$ and $x^{\prime \prime \prime}=x^{\prime}$,

(ii) $\quad x \odot x^{\prime}=0$ and $x \leq x^{\prime} \rightarrow y$.

Let $H$ be a hoop. A non-empty subset $F$ of $H$ is called a filter of $H$ if it satisfies the following assertion,

(F1) $x, y \in F$ implies $x \odot y \in F$,

$(F 2) \quad x \in F$ and $x \leq y$ imply $y \in F$, for any $y \in H$.

Clearly, $1 \in F$, for all filters $F$ of $H$. A filter $F$ of $H$ is called a proper filter if $F \neq H$. It can be easily seen that, if $H$ is a bounded hoop, then a filter is proper if and only if it is not containing 0 (See [15]).

Note. From now on, in this paper, $(H, \odot, \rightarrow, 1)$ or simply $H$ is a hoop and $E$ is a set of parameters, unless otherwise state. 


\section{Soft sub-hoops}

In this section, we introduce the notions of sub-hoop and soft-hoop, and investigate some properties of them.

Definition 3.1. A sub-hoop of a hoop $H$ is a subset $S$ of $H$ containing the unit element of $H$. It means that a subset $S$ of a hoop $H$ is called a sub-hoop of $H$ if $1 \in S, x \odot y \in S$ and $x \rightarrow y \in S$, for all $x, y \in S$.

Example 3.2. ( $i$ ) Every filter $F$ of $H$ is a sub-hoop of $H$.

Because, for any $x, y \in F, x \odot y \in F$ and by Proposition 2.1(iii), $y \leq x \rightarrow y$, since $y \in F$ and $F$ is a filter of $H, x \rightarrow y \in F$.

(ii) Let $H=\{0, a, b, c, d, 1\}$ be a set. Define the operations $\odot$ and $\rightarrow$ on $H$ as follow:

\begin{tabular}{l|cccccc}
$\rightarrow$ & 0 & $\mathrm{a}$ & $\mathrm{b}$ & $\mathrm{c}$ & $\mathrm{d}$ & 1 \\
\hline 0 & 1 & 1 & 1 & 1 & 1 & 1 \\
$\mathrm{a}$ & $\mathrm{c}$ & 1 & $\mathrm{~b}$ & $\mathrm{c}$ & $\mathrm{b}$ & 1 \\
$\mathrm{~b}$ & $\mathrm{~d}$ & $\mathrm{a}$ & 1 & $\mathrm{~b}$ & $\mathrm{a}$ & 1 \\
$\mathrm{c}$ & $\mathrm{a}$ & $\mathrm{a}$ & 1 & 1 & $\mathrm{a}$ & 1 \\
$\mathrm{~d}$ & $\mathrm{~b}$ & 1 & 1 & $\mathrm{~b}$ & 1 & 1 \\
1 & 0 & $\mathrm{a}$ & $\mathrm{b}$ & $\mathrm{c}$ & $\mathrm{d}$ & 1
\end{tabular}

\begin{tabular}{l|llllll}
$\odot$ & 0 & $\mathrm{a}$ & $\mathrm{b}$ & $\mathrm{c}$ & $\mathrm{d}$ & 1 \\
\hline 0 & 0 & 0 & 0 & 0 & 0 & 0 \\
$\mathrm{a}$ & 0 & $\mathrm{a}$ & $\mathrm{d}$ & 0 & $\mathrm{~d}$ & $\mathrm{a}$ \\
$\mathrm{b}$ & 0 & $\mathrm{~d}$ & $\mathrm{c}$ & $\mathrm{c}$ & 0 & $\mathrm{~b}$ \\
$\mathrm{c}$ & 0 & 0 & $\mathrm{c}$ & $\mathrm{c}$ & 0 & $\mathrm{c}$ \\
$\mathrm{d}$ & 0 & $\mathrm{~d}$ & 0 & 0 & 0 & $\mathrm{~d}$ \\
1 & 0 & $\mathrm{a}$ & $\mathrm{b}$ & $\mathrm{c}$ & $\mathrm{d}$ & 1
\end{tabular}

By routine calculations we can see that $(H, \odot, \rightarrow, 0,1)$ is a bounded hoop. Let $S_{1}=\{a, 1\}$ and $S_{2}=\{b, c, 1\}$. It is easy to see that $S_{1}$ and $S_{2}$ are two sub-hoops of $H$.

Proposition 3.3. Let $\left\{H_{i} \mid i \in I\right\}$ be a family of sub-hoops of $H$. Then $\bigcap_{i \in I} H_{i}$ is a sub-hoop of $H$.

Proof. The proof is clear.

In the following example, we show that the union of a family of sub-hoops may not be a sub-hoop, in general.

Example 3.4. Let $H$ be a hoop as in Example 3.2(ii). As we see, $S_{1}$ and $S_{2}$ are two sub-hoops of $H$. But it is clear that $S=S_{1} \cup S_{2}=\{a, b, c, 1\}$ is not a sub-hoop of $H$, because $a \odot b=d \notin S$.

In the following proposition we investigate that under which condition, the union of a family of sub-hoops is a sub-hoop.

Proposition 3.5. Let $\left\{H_{i} \mid i \in I\right\}$ be a family of sub-hoops of $H$. If, for any $i, j \in I, H_{i} \subset H_{j}$ or $H_{j} \subset H_{i}$, then $\bigcup_{i \in I} H_{i}$ is a sub-hoop of $H$. 
Proof. The proof is clear.

Definition 3.6. Let $(\lambda, A)$ be a soft set over $H$, where $A \subseteq E$. Then $(\lambda, A)$ is called a soft hoop of $H$ if $\lambda(e)$ is a sub-hoop of $H$, for any $e \in A$.

The set of all soft hoops over $H$ is denoted by $\operatorname{Soft}_{E}(H)$, that is

$\operatorname{Soft}_{E}(H)=\left\{\left(\lambda_{i}, A_{j}\right) \mid A_{j} \subseteq E, \lambda_{i}: A_{j} \rightarrow \rho(H)\right.$ and $\left(\lambda_{i}, A_{j}\right)$ is a soft hoop of $\left.H\right\}$

Example 3.7. (i) Let $H=\{0, a, b, 1\}$ be a set. Define the operations $\odot$ and $\rightarrow$ on $H$ as follows:

\begin{tabular}{l|llll}
$\rightarrow$ & 0 & $\mathrm{a}$ & $\mathrm{b}$ & 1 \\
\hline 0 & 1 & 1 & 1 & 1 \\
$\mathrm{a}$ & $\mathrm{a}$ & 1 & 1 & 1 \\
$\mathrm{~b}$ & 0 & $\mathrm{a}$ & 1 & 1 \\
1 & 0 & $\mathrm{a}$ & $\mathrm{b}$ & 1
\end{tabular}

\begin{tabular}{l|llll}
$\odot$ & 0 & $\mathrm{a}$ & $\mathrm{b}$ & 1 \\
\hline 0 & 0 & 0 & 0 & 0 \\
$\mathrm{a}$ & 0 & 0 & $\mathrm{a}$ & $\mathrm{a}$ \\
$\mathrm{b}$ & 0 & $\mathrm{a}$ & $\mathrm{b}$ & $\mathrm{b}$ \\
1 & 0 & $\mathrm{a}$ & $\mathrm{b}$ & 1
\end{tabular}

Routine calculations show that $(H, \odot, \rightarrow, 0,1)$ is a bounded hoop. Let $A \subseteq E$ in which $\left\{A_{1}, A_{2}\right\}$ is a partition of $A$. Define a set-valued function as follows:

$$
\lambda: A \rightarrow \rho(H), e \mapsto \begin{cases}\{b, 1\} & \text { if } e \in A_{1}, \\ \{0, a, 1\} & \text { if } e \in A_{2} .\end{cases}
$$

Then $(\lambda, A)$ is a soft hoop over $H$.

(ii) Let $\mu: H \rightarrow[0,1]$ be a map such that, for any $x, y \in H$, we have

$$
\mu(x \odot y) \geq \min \{\mu(x), \mu(y)\} \text { and } \mu(x \rightarrow y) \geq \min \{\mu(x), \mu(y)\} .
$$

For any $\varepsilon \in[0,1]$, consider the set $U(\mu ; \varepsilon)=\{x \in H \mid \mu(x) \geq \varepsilon\}$. Define a map

$$
\lambda:[0,1] \rightarrow \rho(H), \varepsilon \mapsto U(\mu, \varepsilon) .
$$

Then $(\lambda,[0,1])$ is a soft hoop over $H$.

Proposition 3.8. Let $(\lambda, A)$ be a soft hoop of $H$ and $B \subseteq A$. Then $\left(\left.\lambda\right|_{B}, B\right)$ is a soft hoop of $H$, too.

Proof. It is obvious.

In the following example, we show that the converse of Proposition 3.8 may not be true, in general. 
Example 3.9. Let $H=\{0, a, b, c, d, 1\}$ be a bounded hoop as in Example 3.2(ii), then let $A \subseteq E$ such that $\left\{A_{1}, A_{2}\right\}$ be a partition of $A$. Define a set-valued function as follows:

$$
\lambda: A \rightarrow \rho(H), e \mapsto \begin{cases}\{0,1\} & \text { if } e \in A_{1} \\ \{0, c, d, 1\} & \text { if } e \in A_{2} .\end{cases}
$$

Then $\left(\lambda, A_{1}\right)$ is a soft hoop over $H$, but $(\lambda, A)$ is not a soft hoop over $H$. Because if $e \in A_{2}$, then $\lambda(e)=\{0, c, d, 1\}$ is not a sub-hoop of $H$. But if we take $B=A_{1} \subset A$, then $\left(\left.\lambda\right|_{B}, B\right)$ is a soft hoop over $H$.

Theorem 3.10. Let $H$ be a hoop and $\left\{\left(\lambda_{i}, A_{j}\right)\right\}_{i, j \in I} \subseteq \operatorname{Soft}_{E}(H)$. Then the following statements hold:

(i) The extended intersection $\bigcap_{i, j \in I}\left(\lambda_{i}, A_{j}\right)$ is a soft hoop over $H$, if it is non-null.

(ii) The restricted intersection $\prod_{i, j \in I}\left(\lambda_{i}, A_{j}\right)$ is a soft hoop over $H$, if it is non-null.

(iii) The $\bigwedge$-intersection $\bigwedge_{i, j \in I}\left(\lambda_{i}, A_{j}\right)$ is a soft hoop over $H$, if it is non-null.

Proof. (i) As we know, $\bigcup_{j \in I} A_{j} \subset A$ and by Proposition 3.3, $\bigcap_{i \in I} \lambda_{i}$ is a sub-hoop of $H$. So $\bigcap_{i, j \in I}\left(\lambda_{i}, A_{j}\right)$ is a soft hoop over $H$.

The proof of other items is similar to the proof of $(i)$.

In the following example, we show that different kinds of union of a family of soft hoops may not be a soft hoop, in general.

Example 3.11. Let $H$ be a hoop as Example 3.2(ii) and $A=\left\{e_{1}, e_{2}\right\} \subseteq E$ such that $A_{1}=\left\{e_{1}\right\}$ and $A_{2}=\left\{e_{2}\right\}$. Define $\lambda_{1}\left(e_{1}\right)=\{a, 1\}$ and $\lambda_{2}\left(e_{2}\right)=$ $\{b, c, 1\}$. Routine calculations show that $\left(\lambda_{1}, A_{1}\right)$ and $\left(\lambda_{2}, A_{2}\right)$ are two soft hoops over $H$, but $\left(\lambda_{1}, A_{1}\right) \cup\left(\lambda_{2}, A_{2}\right)=\left(\left\{e_{1}, e_{2}\right\},\{a, b, c, 1\}\right)$ is not a soft hoop over $H$. Because $a \odot b=d \notin\{a, b, c, 1\}$.

Definition 3.12. Let $(\lambda, A)$ and $(\gamma, B)$ be two soft hoops over $H$. Then $(\lambda, A)$ is called a soft sub-hoop of $(\gamma, B)$ if it satisfies:

(i) $A \subseteq B$,

(ii) $\lambda(e)$ is a sub-hoop of $\gamma(e)$, for all $e \in A$.

If $(\lambda, A)$ is a soft sub-hoop of $(\gamma, B)$, then we denoted it by $(\lambda, A) \subseteq(\gamma, B)$. Two soft sub-hoops $(\lambda, A)$ and $(\gamma, B)$ are said to be a soft equal hoop, denoted by $(\lambda, A)=(\gamma, B)$, if $(\lambda, A) \subseteq(\gamma, B)$ and $(\gamma, B) \subseteq(\lambda, A)$. 
Example 3.13. Let $H$ be a hoop as Example 3.2 and $E=\left\{e_{1}, e_{2}, e_{3}\right\}$. Define

$$
A=\left\{e_{1}\right\}, \gamma\left(e_{1}\right)=\{1, a\}, B=\left\{e_{1}, e_{2}\right\}, \lambda(e)= \begin{cases}\{0, a, c, 1\} & \text { if } e=e_{1}, \\ \{c, 1\} & \text { if } e=e_{2} .\end{cases}
$$

It is clear that $(\gamma, A) \subseteq(\lambda, B)$.

Proposition 3.14. ( $i)$ If $(\lambda, A)$ and $(\gamma, A)$ are two soft hoops over $H$ such that $\lambda(e)$ is a sub-hoop of $\gamma(e)$, for all $e \in A$, then $(\lambda, A)$ is a soft sub-hoop of $(\gamma, A)$.

(ii) If $(\lambda, H)$ and $(\varphi,\{1\})$ are two soft hoops over $H$, then $(\varphi,\{1\})$ is a soft sub-hoop of $(\lambda, H)$.

Proof. It is straightforward.

\section{Algebraic structures on $\operatorname{Soft}_{E}(H)$}

In this section, we introduce some operations on $\operatorname{Soft}_{E}(H)$ and we show that $\operatorname{Soft}_{E}(H)$ with these operations is a Heyting algebra and a hoop. Also, we define a congruence relation on $\operatorname{Soft}_{E}(H)$ and investigate the quotient that is made by it.

Note. From now on, in this section, for any $e \in A, \lambda(e) \tilde{\simeq} \gamma(e)$ means that $\lambda(e)$ is a sub-hoop of $\gamma(e)$.

Theorem 4.1. $\left(\operatorname{Soft}_{E}(H), \subseteq\right)$ is a poset.

Proof. Let $(\lambda, A) \in \operatorname{Soft}_{E}(H)$. Since $A \subseteq A$ and for any $e \in A, \lambda(e) \tilde{\subseteq} \lambda(e)$, by Definition 3.12, we have $(\lambda, A) \subseteq(\lambda, A)$. Hence, $\subseteq$ is reflexive.

Now, let $(\lambda, A),(\gamma, B) \in \operatorname{Soft}_{E}(H)$, such that $(\lambda, A) \subseteq(\gamma, B)$ and $(\gamma, B) \subseteq$ $(\lambda, A)$. Then $A \subseteq B$ and, for any $e \in A, \lambda(e) \subseteq \tilde{\subseteq} \gamma(e)$. Also, $B \subseteq A$ and for any $e \in$ $B, \gamma(e) \subseteq \tilde{\subseteq}(e)$. Hence, $(\lambda, A)=(\gamma, B)$, and so $\subseteq$ is antisymmetry. Now, we prove that the relation $\subseteq$ is transitive. Let $(\lambda, A),(\gamma, B),(\eta, C) \in \operatorname{Soft}_{E}(H)$, such that $(\lambda, A) \subseteq(\gamma, B)$ and $(\gamma, B) \subseteq(\eta, C)$. Then $A \subseteq B$ and, for any $e \in A, \lambda(e) \tilde{\tilde{\subseteq}} \gamma(e)$ and $B \subseteq C$ and, for any $e \in B, \gamma(e) \tilde{\subseteq} \eta(e)$. It is easy to see that $A \subseteq C$ and $\lambda(e) \stackrel{\tilde{\subseteq}}{\eta}(e)$, for any $e \in A$. Then $(\lambda, A) \subseteq(\eta, C)$, and so $\left(\operatorname{Soft}_{E}(H), \subseteq\right.$ ) is a poset.

Note. In the following example, we show that extended union of the family of soft hoops is not a soft hoop, in general. So, from now on, in this section, we suppose that $\operatorname{Soft}_{E}(H)$ is closed under two operations $\prod$ and $\amalg$. 
Example 4.2. Let $H$ be a bounded hoop as in Example 3.2(ii). Suppose $E=\left\{e_{1}, e_{2}, e_{3}, e_{4}\right\}$ and define

$$
\begin{gathered}
A_{1}=\left\{e_{1}, e_{2}, e_{3}, e_{4}\right\}, \lambda_{1}(e)= \begin{cases}\{1\} & \text { if } e=e_{1}, \\
\{\mathrm{a}, 1\} & \text { if } e=e_{2}, \\
\{0,1\} & \text { if } e \in\left\{e_{3}, e_{4}\right\}\end{cases} \\
A_{2}=\left\{e_{1}, e_{2}, e_{3}\right\}, \lambda_{2}(e)= \begin{cases}\{1, \mathrm{c}\} & \text { if } e=e_{3}, \\
\{1, \mathrm{~b}, \mathrm{c}\} & \text { if } e \in\left\{e_{1}, e_{2}\right\}\end{cases} \\
A_{3}=\left\{e_{1}, e_{2}, e_{3}, e_{4}\right\}, \lambda_{3}(e)= \begin{cases}\mathrm{H} & \text { if } e=e_{1}, \\
\{0, \mathrm{a}, \mathrm{c}, 1\} & \text { if } e=e_{4}, \\
\{1\} & \text { if } e \in\left\{e_{3}, e_{2}\right\}\end{cases} \\
A_{4}=\left\{e_{2}, e_{3}\right\}, \lambda_{4}(e)= \begin{cases}\{0,1\} & \text { if } e=e_{3}, \\
\{\mathrm{a}, 1\} & \text { if } e=e_{2} .\end{cases}
\end{gathered}
$$

By routine calculation, it is clear that $\left(\lambda_{i}, A_{i}\right)$, for $1 \leq i \leq 4$, are soft hoops of $H$. But $(\gamma, B)=\coprod_{i=1}^{4}\left(\lambda_{i}, A_{i}\right)$ is not a soft hoop of $H$. Because, for $e=e_{3}$, we have $\gamma(e)=\{0, c, 1\}$, which is not a sub hoop of $H$, since $c \rightarrow 0=a \notin \gamma(e)$.

Definition 4.3. A soft set $(\lambda, A)$ over $H$ is called a trivial soft hoop (resp., whole soft hoop) if it is a soft hoop over $H$ and satisfies the condition $\lambda(e)=\{1\}$ (resp., $\lambda(e)=H$ ), for all $e \in A$.

Theorem 4.4. $\left(\operatorname{Soft}_{E}(H), \prod, \amalg, H_{E}, \emptyset_{\emptyset}\right)$ is a complete distributive bounded lattice, where $H_{E}=(H, E)$ is the whole soft hoop over $H$ and $\emptyset_{\emptyset}=(\emptyset, \emptyset)$ is a null soft hoop over $H$.

Proof. By Theorem 4.1, $\left(\operatorname{Soft}_{E}(H), \subseteq\right)$ is a poset. Also, we have

$$
\emptyset_{\emptyset} \subseteq \emptyset_{A} \subseteq(\lambda, A) \subseteq H_{A} \subseteq H_{E}
$$

Hence, $\emptyset_{\emptyset}$ and $H_{E}$ are the smallest and the greatest elements of $\operatorname{Soft}_{E}(H)$, respectively, and so $\operatorname{Soft}_{E}(H)$ is bounded.

Now, we prove that $\left(\operatorname{Soft}_{E}(H), \prod, \amalg, H_{E}, \emptyset_{\emptyset}\right)$ is a lattice. Let $(\lambda, A),(\gamma, B),(\eta, C) \in$ $\operatorname{Soft}_{H}(E)$. Then it is clear that $(\lambda, A) \amalg(\lambda, A)=(\lambda, A)$. Also,

$$
(\lambda, A) \coprod(\gamma, B)=(\lambda \cup \gamma, A \cup B)=(\gamma \cup \lambda, B \cup A)=(\gamma, B) \coprod(\lambda, A)
$$


Moreover, we have

$$
\begin{aligned}
(\lambda, A) \coprod((\gamma, B) \coprod(\eta, C)) & =(\lambda, A) \coprod(\gamma \cup \eta, B \cup C) \\
& =(\lambda \cup(\gamma \cup \eta), A \cup(B \cup C)) \\
& =((\lambda \cup \gamma) \cup \eta,(A \cup B) \cup C) \\
& =(\lambda \cup \gamma, A \cup B) \coprod(\eta, C) \\
& =((\lambda, A) \coprod(\gamma, B)) \coprod(\eta, C)
\end{aligned}
$$

Finally,

$$
\begin{aligned}
(\lambda, A) \prod((\lambda, A) \coprod(\gamma, B)) & =(\lambda, A) \prod(\lambda \cup \gamma, A \cup B) \\
& =(\lambda \cap(\lambda \cup \gamma), A \cap(A \cup B)) \\
& =(\lambda, A)
\end{aligned}
$$

By the similar way, we can proof all these conditions for the operation $\prod$. Hence, $\left(\operatorname{Soft}_{E}(H), \prod, \coprod\right)$ is a lattice. Also, it is clear that $\left(\operatorname{Soft}_{E}(H), \prod, \coprod\right)$ is a complete lattice.

So, it is enough to prove that $\left.\operatorname{Soft}_{E}(H), \Pi, \coprod\right)$, is a distributive lattice. For this, we have

$$
\begin{aligned}
(\lambda, A) \prod[(\gamma, B) \coprod(\eta, C)] & =(\lambda, A) \prod(\gamma \cup \eta, B \cup C) \\
& =[\lambda \cap(\gamma \cup \eta), A \cap(B \cup C)] \\
& =[(\lambda \cap \gamma) \cup(\lambda \cap \eta),(A \cap B) \cup(A \cap C)] \\
& =(\lambda \cap \gamma, A \cap B) \coprod(\lambda \cap \eta, A \cap C) \\
& =\left[(\lambda, A) \prod(\gamma, B)\right] \coprod\left[(\lambda, A) \prod(\eta, C)\right]
\end{aligned}
$$

The proof of other case is similar. Therefore, $\left(\operatorname{Soft}_{E}(H), \prod, \coprod, H_{E}, \emptyset_{\emptyset}\right)$ is a complete distributive bounded lattice.

Let $(\lambda, A),(\gamma, B) \in \operatorname{Soft}_{E}(H)$. Then we define the operation $\rightarrow$ on

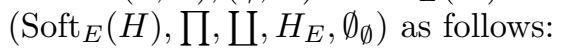

$$
(\lambda, A) \rightarrow(\gamma, B)=\coprod_{i, j \in I}\left\{\left(\lambda_{i}, A_{j}\right) \mid(\lambda, A) \prod\left(\lambda_{i}, A_{j}\right) \subseteq(\gamma, B)\right\}
$$

Proposition 4.5. Let $(\lambda, A),(\gamma, B) \in$ Soft $_{E}(H)$. Then the following assertions, hold:

(i) $(\lambda, A) \rightarrow(\lambda, A)=H_{E}$.

(ii) $(\lambda, A) \subseteq(\gamma, B)$ if and only if $(\lambda, A) \rightarrow(\gamma, B)=H_{E}$. 
Proof. (i) Let $(\lambda, A),(\gamma, B) \in \operatorname{Soft}_{E}(H)$. Then,

$$
\begin{aligned}
& (\lambda, A) \rightarrow(\lambda, A) \\
= & \coprod_{i, j \in I}\left\{\left(\lambda_{i}, A_{j}\right) \mid(\lambda, A) \prod\left(\lambda_{i}, A_{j}\right) \subseteq(\lambda, A)\right\} \\
= & \coprod_{i, j \in I}\left\{\left(\lambda_{i}, A_{j}\right) \mid\left(\lambda \cap \lambda_{i}, A \cap A_{j}\right) \subseteq(\lambda, A)\right\} \\
= & \coprod_{i, j \in I}\left\{\left(\lambda_{i}, A_{j}\right) \mid A \cap A_{j} \subseteq A \text { and }\left(\lambda \cap \lambda_{i}\right)(e) \tilde{\subseteq} \lambda(e), \text { for any } e \in A \cap A_{j}\right\} \\
= & \left(\bigcup_{i \in I} \lambda_{i}, \bigcup_{j \in I} A_{j}\right) \\
= & H_{E}
\end{aligned}
$$

(ii) Let $(\lambda, A) \subseteq(\gamma, B)$. Then by Definition 3.12, $A \subseteq B$ and $\lambda(e) \tilde{\subseteq} \gamma(e)$, for any $e \in A$. Thus,

$$
\begin{aligned}
(\lambda, A) \rightarrow(\gamma, B) & =\coprod_{i, j \in I}\left\{\left(\lambda_{i}, A_{j}\right) \mid(\lambda, A) \prod\left(\lambda_{i}, A_{j}\right) \subseteq(\gamma, B)\right\} \\
& =\coprod_{i, j \in I}\left\{\left(\lambda_{i}, A_{j}\right) \mid\left(\lambda \cap \lambda_{i}, A \cap A_{j}\right) \subseteq(\gamma, B)\right\} \\
& =H_{E}
\end{aligned}
$$

Since $A \cap A_{j} \subseteq B$ and $\left(\lambda \cap \lambda_{i}\right)(e) \tilde{\tilde{\subseteq}} \gamma(e)$, for any $e \in A \cap A_{j}$.

Conversely, assume that $(\lambda, A) \rightarrow(\gamma, B)=H_{E}$. Then, for any $(\eta, C) \in$ $\operatorname{Soft}_{E}(H)$ we have, $(\lambda, A) \prod(\eta, C) \subseteq(\gamma, B)$. Hence, for $(\eta, C)=(\lambda, A)$, we have $(\lambda, A) \subseteq(\gamma, B)$.

Theorem 4.6. $\left(\operatorname{Soft}_{E}(H), \prod, \rightarrow, H_{E}, \emptyset_{\emptyset}\right)$ is a bounded hoop.

Proof. The operation $\prod$ will be consider as a product operation on $\operatorname{Soft}_{E}(H)$. So

$$
(\lambda, A) \prod(\gamma, B)=(\lambda \cap \gamma, A \cap B)
$$

Then it is clear that $\operatorname{Soft}_{E}(H)$ is closed with respect to $\prod$. Now, we prove that $\left(\operatorname{Soft}_{E}(H), \prod, H_{E}\right)$ is a commutative monoid with the greatest element. 
For this, for any $(\lambda, A),(\gamma, B),(\eta, C) \in \operatorname{Soft}_{E}(H)$, we have,

$$
\begin{aligned}
(\lambda, A) \prod\left\{(\gamma, B) \prod(\eta, C)\right\} & =(\lambda, A) \prod(\gamma \cap \eta, B \cap C) \\
& =(\lambda \cap(\gamma \cap \eta), A \cap(B \cap C)) \\
& =((\lambda \cap \gamma) \cap \eta,(A \cap B) \cap C) \\
& =(\lambda \cap \gamma, A \cap B) \prod(\eta, C) \\
& =\left\{(\lambda, A) \prod(\gamma, B)\right\} \prod(\eta, C)
\end{aligned}
$$

and

$$
(\lambda, A) \prod H_{E}=(\lambda, A) \prod(H, E)=(\lambda \cap H, A \cap E)=(\lambda, A)
$$

Therefore, $\left(\operatorname{Soft}_{E}(H), \prod, H_{E}\right)$ is a commutative monoid with the greatest element $H_{E}$. By Proposition 4.5(i), $(\lambda, A) \rightarrow(\lambda, A)=H_{E}$,

$$
\begin{aligned}
& =(\lambda, A) \rightarrow((\gamma, B) \rightarrow(\eta, C)) \\
& =\coprod_{i, j \in I}\left\{\left(\lambda_{i}, A_{j}\right) \mid\left(\lambda_{i}, A_{j}\right) \cap(\lambda, A) \subseteq \coprod_{k, l \in I}\left\{\left(\eta_{k}, C_{l}\right) \mid(\gamma, B) \prod\left(\eta_{k}, C_{l}\right) \subseteq(\eta, C)\right\}\right\}
\end{aligned}
$$

So we have, for every $i, j \in I,\left(\lambda_{i}, A_{j}\right) \cap(\lambda, A) \subseteq \coprod_{k, l \in I}\left\{\left(\eta_{k}, C_{l}\right)\right\}$. Then,

$$
\left(\lambda_{i}, A_{j}\right) \cap(\lambda, A) \cap(\gamma, B) \subseteq \coprod_{k, l \in I}\left\{\left(\eta_{k}, C_{l}\right) \cap(\gamma, B)\right\} .
$$

Since $\left(\operatorname{Soft}_{E}(H), \prod, \amalg\right)$ is a distributive lattice, we get that

$$
\left(\lambda_{i}, A_{j}\right) \cap(\lambda, A) \cap(\gamma, B) \subseteq \coprod_{k, l \in I}\left\{\left(\eta_{k}, C_{l}\right) \cap(\gamma, B)\right\} \subseteq(\eta, C) .
$$

Therefore,

$$
\begin{aligned}
& (\lambda, A) \rightarrow((\gamma, B) \rightarrow(\eta, C)) \\
= & \coprod_{i, j \in I}\left\{\left(\lambda_{i}, A_{j}\right) \mid\left(\lambda_{i}, A_{j}\right) \cap(\lambda, A) \cap(\gamma, B) \subseteq(\eta, C)\right\} \\
= & ((\lambda, A) \cap(\gamma, B)) \rightarrow(\eta, C)
\end{aligned}
$$

Now, it is enough to prove that,

$$
(\lambda, A) \prod(\gamma, B)=(\lambda, A) \prod((\lambda, A) \rightarrow(\gamma, B))
$$

So, by Theorem 4.4 , since $(\lambda, A) \prod(\gamma, B)=(\gamma, B) \prod(\lambda, A)$, we consequence that,

$$
\begin{aligned}
(\lambda, A) \prod((\lambda, A) \rightarrow(\gamma, B)) & =(\lambda, A) \prod(\gamma, B)=(\gamma, B) \prod(\lambda, A) \\
& =(\gamma, B) \prod((\gamma, B) \rightarrow(\lambda, A))
\end{aligned}
$$


Thus, according to definition $\rightarrow$, it is clear that,

$$
(\gamma, B) \subseteq \coprod_{i, j \in I}\left\{\left(\lambda_{i}, A_{j}\right) \mid(\lambda, A) \prod\left(\lambda_{i}, A_{j}\right) \subseteq(\gamma, B)\right\}=(\lambda, A) \rightarrow(\gamma, B)
$$

Then

$$
(\lambda, A) \prod(\gamma, B) \subseteq(\lambda, A) \prod((\lambda, A) \rightarrow(\gamma, B))
$$

Conversely,

$$
\begin{aligned}
& (\lambda, A) \prod((\lambda, A) \rightarrow(\gamma, B)) \\
= & (\lambda, A) \prod\left(\coprod_{i, j \in I}\left\{\left(\lambda_{i}, A_{j}\right) \mid(\lambda, A) \prod\left(\lambda_{i}, A_{j}\right) \subseteq(\gamma, B)\right\}\right) \\
= & \coprod_{i, j \in I}\left\{\left(\lambda_{i} \cap \lambda, A_{j} \cap A\right) \mid\left(\lambda \cap \lambda_{i}, A \cap A_{j}\right) \subseteq(\gamma, B)\right\}
\end{aligned}
$$

Since $\left(\lambda \cap \lambda_{i}\right)(e) \tilde{\widetilde{\subseteq}} \gamma(e)$, we have $\left(\lambda \cap \lambda \cap \lambda_{i}\right)(e) \tilde{\subseteq}(\lambda \cap \gamma)(e)$, for any $e \in A \cap A_{i}$. Also, from $A \cap A_{j} \subseteq B$, we get that $A \cap A \cap A_{i} \subseteq A \cap B$. So,

$$
\coprod_{i \in I}\left(\lambda \cap \lambda_{i}\right)(e) \tilde{\simeq}(\lambda \cap \gamma)(e), \bigcup_{j \in I}\left(A \cap A_{j}\right) \subseteq A \cap B
$$

Hence,

$$
\coprod_{i, j \in I}\left(\lambda \cap \lambda_{i}, A \cap A_{j}\right) \subseteq(\lambda \cap \gamma, A \cap B)
$$

Finally,

$$
\begin{aligned}
& (\lambda, A) \prod((\lambda, A) \rightarrow(\gamma, B)) \\
= & (\lambda, A) \prod\left(\coprod_{i, j \in I}\left\{\left(\lambda_{i}, A_{j}\right) \mid(\lambda, A) \prod\left(\lambda_{i}, A_{j}\right) \subseteq(\gamma, B)\right\}\right) \\
\subseteq & (\lambda, A) \prod(\gamma, B)
\end{aligned}
$$

Therefore, $\left(\operatorname{Soft}_{E}(H), \prod, \rightarrow, H_{E}, \emptyset_{\emptyset}\right)$ is a bounded hoop.

Definition 4.7. [6] A Heyting algebra is an algebra $(A, \vee, \wedge, \rightarrow, 1)$ of type $(2,2,2,0)$, where $(A, \vee, \wedge, 1)$ is a lattice with the greatest element 1 and, for any $x, y, z \in A$,

$$
x \leq y \rightarrow z \text { if and only if } x \wedge y \leq z
$$

Theorem 4.8. $\left(\operatorname{Soft}_{E}(H), \Pi, \coprod, \rightarrow, H_{E}\right)$ is a Heyting algebra. 
Proof. By Theorem 4.4, we prove that $\left(\operatorname{Soft}_{E}(H), \prod, \amalg, H_{E}, \emptyset_{\emptyset}\right)$ is a distributive lattice with the greatest element $H_{E}$. Now, it is enough to prove that the condition $(*)$ hold. Let $(\lambda, A),(\gamma, B)$ and $(\eta, C) \in \operatorname{Soft}_{E}(H)$ such that $(\lambda, A) \subseteq(\gamma, B) \rightarrow(\eta, C)$. By definition of the operation $\rightarrow$, we have

$$
(\gamma, B) \rightarrow(\eta, C)=\coprod_{i, j \in I}\left\{\left(\mu_{i}, D_{j}\right) \mid\left(\mu_{i}, D_{j}\right) \cap(\gamma, B) \subseteq(\eta, C)\right\}=\coprod_{i, j \in I}\left(\mu_{i}, D_{j}\right)
$$

Since $(\lambda, A) \subseteq(\gamma, B) \rightarrow(\eta, C)$, we get that $(\lambda, A) \subseteq \coprod_{i, j \in I}\left(\mu_{i}, D_{j}\right)$. Then

$$
(\lambda, A) \prod(\gamma, B) \subseteq(\gamma, B) \prod\left(\coprod_{i, j \in I}\left(\mu_{i}, D_{j}\right)\right)
$$

Moreover, since the lattice is distributive, we consequence that

$$
\left.(\lambda, A) \prod(\gamma, B) \subseteq \coprod_{i, j \in I}\left[\left(\mu_{i}, D_{j}\right) \prod(\gamma, B)\right)\right] \subseteq \coprod(\eta, C)=(\eta, C)
$$

So, $(\lambda, A) \prod(\gamma, B) \subseteq(\eta, C)$.

Conversely, suppose $(\lambda, A) \prod(\gamma, B) \subseteq(\eta, C)$. Then, according to definition of $\rightarrow$, we consequence that $(\lambda, A) \in(\gamma, B) \rightarrow(\eta, C)$. Hence, $(\lambda, A) \subseteq(\gamma, B) \rightarrow$ $(\eta, C)$. Therefore, $\left(\operatorname{Soft}_{E}(H), \prod, \amalg, \rightarrow, H_{E}\right)$ is a Heyting algebra.

As we know, by $\left(\rightarrow, \emptyset_{\emptyset}\right)$ we can define

$$
(\lambda, A) \rightarrow \emptyset_{\emptyset}=\coprod_{i, j \in I}\left\{\left(\lambda_{i}, A_{j}\right) \mid\left(\left(\lambda_{i}, A_{j}\right) \cap(\lambda, A) \subseteq(\emptyset, \emptyset)\right\} .\right.
$$

Since for any $\emptyset_{\emptyset} \neq\left(\lambda_{i}, A_{j}\right) \in \operatorname{Soft}_{E}(H), 1 \in \lambda_{i}$, then $\left(\lambda_{i}, A_{j}\right) \cap(\lambda, A) \neq$ $(\emptyset, \emptyset)$. Hence, $(\lambda, A) \rightarrow \emptyset_{\emptyset}=\emptyset_{\emptyset}$ Therefore, we conclude that for any $(\lambda, A) \in$ $\operatorname{Soft}_{E}(H),(\lambda, A) \rightarrow \emptyset_{\emptyset}=\emptyset_{\emptyset}$.

Example 4.9. Let $H$ be a hoop in Example 3.7 and $E=\left\{e_{1}, e_{2}\right\}$. Then define the set of soft hoops as follows:

$A_{1}=\emptyset$ and $\lambda_{1}: A \rightarrow \rho(H)$ such that $\lambda_{1}=\emptyset$ so $\left(\lambda_{1}, A_{1}\right)=(\emptyset, \emptyset)=\emptyset_{\emptyset}$,

$A_{2}=E$ and $\lambda_{2}: E \rightarrow \rho(H)$ such that $\lambda_{2}=H$, so $\left(\lambda_{2}, A_{2}\right)=H_{E}$,

$A_{3}=\left\{e_{1}\right\}$ and $\lambda_{3}: A_{3} \rightarrow \rho(H)$ such that $\lambda_{3}\left(e_{1}\right)=\{0,1\}$,

$A_{4}=\left\{e_{1}, e_{2}\right\}$ and $\lambda_{4}: A_{4} \rightarrow \rho(H)$ such that $\lambda_{4}\left(e_{1}\right)=\lambda_{4}\left(e_{2}\right)=\{0, a, 1\}$,

$A_{5}=\left\{e_{1}, e_{3}\right\}$ and $\lambda_{5}: A_{5} \rightarrow \rho(H)$ such that $\lambda_{5}(e)=\left\{\begin{array}{ll}\{b, 1\} & \text { if } e=e_{1}, \\ \{0, b, 1\} & \text { if } e=e_{3} .\end{array}\right.$.

Then by routine calculations, we can see that, for any $2 \leq i \leq 5$,

$$
\left(\lambda_{i}, A_{i}\right) \rightarrow \emptyset_{\emptyset}=\emptyset_{\emptyset} \text { and }\left(\lambda_{1}, A_{1}\right) \rightarrow \emptyset_{\emptyset}=\emptyset_{\emptyset} \rightarrow \emptyset_{\emptyset}=H_{E}
$$

Hence, it is clear that double negation property does not hold, and so $\operatorname{Soft}_{E}(H), \prod, \rightarrow$ $\left., H_{E}, \emptyset_{\emptyset}\right)$ is not an $M V$-algebra. 
Theorem 4.10. Let $(\lambda, A),(\gamma, B) \in \operatorname{Soft}_{E}(H)$. Define the relation $\equiv$ on Soft $_{E}(H)$ as follows:

$$
(\lambda, A) \equiv(\gamma, B) \Longleftrightarrow \exists(\varphi, C) \in \operatorname{Soft}_{E}(H) \text { st. }(\lambda, A) \prod(\varphi, C) \subseteq(\gamma, B)
$$

Then $\equiv i$ s a congruence relation on $\operatorname{Soft}_{E}(H)$.

Proof. It is clear that $(\lambda, A) \equiv(\lambda, A)$. If $(\lambda, A) \equiv(\gamma, B)$, then there exists $(\varphi, C)=(\gamma, B) \in \operatorname{Soft}_{E}(H)$ such that $(\lambda, A) \prod(\gamma, B) \subseteq(\gamma, B)$. By the similar way, $(\gamma, B) \prod(\lambda, A) \subseteq(\lambda, A)$, and so $(\gamma, B) \equiv(\lambda, A)$. Hence, it is symmetric. Now, let $(\lambda, A) \equiv(\gamma, B)$ and $(\gamma, B) \equiv(\triangle, D)$. Since $(\lambda, A) \equiv(\gamma, B)$, there exists $\left(\varphi_{1}, C_{1}\right) \in \operatorname{Soft}_{E}(H)$ such that,

$$
(\lambda, A) \prod\left(\varphi_{1}, C_{1}\right) \subseteq(\gamma, B)
$$

Also, from $(\gamma, B) \equiv(\triangle, D)$, there exists $\left(\varphi_{2}, C_{2}\right) \in \operatorname{Soft}_{E}(H)$ such that,

$$
(\gamma, B) \prod\left(\varphi_{2}, C_{2}\right) \subseteq(\triangle, D)
$$

Then there exists $\left(\varphi_{1}, C_{1}\right) \prod\left(\varphi_{2}, C_{2}\right) \in \operatorname{Soft}_{E}(H)$ such that

$$
(\lambda, A) \prod\left(\varphi_{1}, C_{1}\right) \prod\left(\varphi_{2}, C_{2}\right) \subseteq(\gamma, B) \prod\left(\varphi_{2}, C_{2}\right) \subseteq(\triangle, D)
$$

Hence, $(\lambda, A) \equiv(\triangle, D)$.

Now, let $(\lambda, A) \equiv(\gamma, B)$. Then there exists $(\varphi, C) \in \operatorname{Soft}_{E}(H)$ such that, $(\lambda, A) \prod(\varphi, C) \subseteq(\gamma, B)$. Since $\prod$ is the product operation on $\operatorname{Soft}_{E}(H)$, for any $(\triangle, D) \in \operatorname{Soft}_{E}(H)$, we get that,

$$
(\lambda, A) \prod(\varphi, C) \prod(\triangle, D) \subseteq(\gamma, B) \prod(\triangle, D)
$$

Thus, there exists $(\varphi, C) \in \operatorname{Soft}_{E}(H)$ such that,

$$
\left((\lambda, A) \prod(\triangle, D)\right) \prod(\varphi, C) \subseteq(\gamma, B) \prod(\triangle, D)
$$

Hence,

$$
(\lambda, A) \prod(\triangle, D) \equiv(\gamma, B) \prod(\triangle, D)
$$

Moreover, suppose $(\lambda, A) \equiv(\gamma, B)$. Then there exists $(\varphi, C)=(\lambda, A) \prod(\gamma, B) \in$ $\operatorname{Soft}_{E}(H)$, such that $(\lambda, A) \prod(\varphi, C) \subseteq(\gamma, B)$. Let $(\triangle, D) \in \operatorname{Soft}_{E}(H)$. Then

$$
((\lambda, A) \rightarrow(\triangle, D)) \prod(\varphi, C)=\coprod_{i, j \in I}\left\{\left(\lambda_{i}, A_{j}\right) \mid(\lambda, A) \prod\left(\lambda_{i}, A_{j}\right) \subseteq(\triangle, D)\right\} \prod(\varphi, C)
$$


Since $\left(\operatorname{Soft}_{E}(H), \prod, \amalg\right)$ is a distributive lattice, and $(\lambda, A) \prod(\varphi, C) \subseteq(\gamma, B)$. By considering $\left(\lambda_{i}, A_{j}\right) \prod(\lambda, A)=\left(\eta_{i}, F_{j}\right)$ we have,

$$
\begin{aligned}
& \coprod_{i, j \in I}\left\{\left(\lambda_{i}, A_{j}\right) \mid(\lambda, A) \prod\left(\lambda_{i}, A_{j}\right) \subseteq(\triangle, D)\right\} \prod(\varphi, C) \\
= & \coprod_{i, j \in I}\left\{\left(\lambda_{i}, A_{j}\right) \prod(\varphi, C) \mid(\lambda, A) \prod\left(\lambda_{i}, A_{j}\right) \prod(\varphi, C) \subseteq(\triangle, D) \prod(\varphi, C) \subseteq(\triangle, D)\right\} \\
= & \coprod_{i, j \in I}\left\{\left(\lambda_{i}, A_{j}\right) \prod(\lambda, A) \prod(\gamma, B) \mid\left(\lambda_{i}, A_{j}\right) \prod(\lambda, A) \prod(\gamma, B) \subseteq(\triangle, D)\right\} \\
\subseteq & \coprod_{i, j \in I}\left\{\left(\lambda_{i}, A_{j}\right) \prod(\lambda, A) \mid\left(\lambda_{i}, A_{j}\right) \prod(\lambda, A) \prod(\gamma, B) \subseteq(\triangle, D)\right\} \\
= & \coprod_{i, j \in I}\left\{\left(\eta_{i}, F_{j}\right) \mid(\gamma, B) \prod\left(\eta_{i}, F_{j}\right) \subseteq(\triangle, D)\right\} \\
= & (\gamma, B) \rightarrow(\triangle, D)
\end{aligned}
$$

Hence, $(\lambda, A) \rightarrow(\triangle, D) \equiv(\gamma, B) \rightarrow(\triangle, D)$.

Now, let $(\lambda, A) \equiv(\gamma, B)$. Then there exists $(\varphi, C) \in \operatorname{Soft}_{E}(H)$, such that $(\lambda, A) \prod(\varphi, C) \subseteq(\gamma, B)$. Suppose $(\delta, G) \in \operatorname{Soft}_{E}(H)$. Thus,

$$
((\delta, G) \rightarrow(\lambda, A)) \prod(\varphi, C)=\coprod_{i, j \in I}\left\{\left(\delta_{i}, G_{j}\right) \mid\left(\delta_{i}, G_{j}\right) \prod(\delta, G) \subseteq(\lambda, A)\right\} \prod(\varphi, C)
$$

Since $\left(\operatorname{Soft}_{E}(H), \prod, \amalg\right)$ is a distributive lattice, $(\lambda, A) \prod(\varphi, C) \subseteq(\gamma, B)$. By considering $\left(\delta_{i}, G_{j}\right) \prod(\varphi, C)=\left(\eta_{i}, F_{j}\right)$ we have

$$
\begin{aligned}
& \coprod_{i, j \in I}\left\{\left(\delta_{i}, G_{j}\right) \mid\left(\delta_{i}, G_{j}\right) \prod(\delta, G) \subseteq(\lambda, A)\right\} \prod(\varphi, C) \\
= & \coprod_{i, j \in I}\left\{\left(\delta_{i}, G_{j}\right) \prod(\varphi, C) \mid\left(\delta_{i}, G_{j}\right) \prod(\varphi, C) \prod(\delta, G) \subseteq(\lambda, A) \prod(\varphi, C)\right\} \\
\subseteq & \coprod_{i, j \in I}\left\{\left(\delta_{i}, G_{j}\right) \prod(\varphi, C) \mid\left(\delta_{i}, G_{j}\right) \prod(\varphi, C) \prod(\delta, G) \subseteq(\lambda, A) \prod(\varphi, C) \subseteq(\gamma, B)\right\} \\
= & \coprod_{i, j \in I}\left\{\left(\eta_{i}, F_{j}\right) \mid(\delta, G) \prod\left(\eta_{i}, F_{j}\right) \subseteq(\gamma, B)\right\} \\
= & (\delta, G) \rightarrow(\gamma, B)
\end{aligned}
$$

Then $(\delta, G) \rightarrow(\gamma, B) \equiv(\delta, G) \rightarrow(\lambda, A)$. Therefore, $\equiv$ is a congruence relation on $\operatorname{Soft}_{E}(H)$.

Corollary 4.11. $\left(\operatorname{Soft}_{E}(H) / \equiv, \prod, \rightarrow, H_{E}\right)$ is a hoop. 


\section{Conclusions and future works}

In this paper, the concept of soft hoop is introduced and some examples are provided. Then, different types of intersections and unions of the family of soft hoops are established. We defined two operations $\odot$ and $\rightarrow$ on a hoop $H$ and a parameters set $E$ and we proved that the set of all soft hoops with these operations is a hoop and also is a Heyting algebra. Finally we introduced a congruence relation on the set of all soft hoops and we investigated the quotient of it. For the future works, it can be investigated that under which conditions, the set of all soft hoops can be considered as one of other algebraic structures.

\section{References}

[1] U. Acar, F. Koyuncu, B. Tanay, Soft sets and soft rings, Computers and Mathematics with Applications, 59 (2010), 3458-3463.

[2] P. Aglianó, I. M. A. Ferreirim, F. Montagna, Basic hoops: an algebraic study of continuous t-norm, 87(1) (2007), 73-98.

[3] H. Aktas, N. Cagman, Soft sets and soft groups, Information Sciences, 177 (2007), 2726-2735.

[4] S. Z. Alavi, R. A. Borzooei, M. Aaly Kologani, Fuzzy filters in pseudo hoops, Journal of Intelligent and Fuzzy Systems, 32 (2017), 1997-2007.

[5] S. Z. Alavi, R. A. Borzooei, M. Aaly Kologani, Filter theory of pseudo hoop algebras, Italian Journal of Pure and Applied Mathematics, 37 (2017 ), 619-632.

[6] V. Boicescu, A. Filipoiu, G, Georgescu, S. Rudeano, Lukasiewics-Moisil algebras, Annals of Discrete Mathematics, 49 (1991), 2-15.

[7] R. A. Borzooei, M. Aaly Kologani, Filter theory of hoop-algebras, Journal of Advanced Research in Pure Mathematics, 1 (2014), 1-15.

[8] R. A. Borzooei, M. Aaly Kologani, Local and perfect semihoops, Journal of Intelligent and Fuzzy Systems, 29 (2015), 223-234.

[9] R. A. Borzooei, M. Aaly Kologani, On fuzzy filters of hoop algebras, The Journal of Fuzzy Mathematics, 25(1) (2017), 177-195.

[10] R. A. Borzooei, M. Aaly Kologani, O. Zahiri, State of hoops, Mathematica Slovaca, 67(1) (2017), 1-16. 
[11] B. Bosbach, Komplementäre halbgruppen. axiomatik und arithmetik, Fundamenta Mathematicae, 64 (1969), 257-287.

[12] B. Bosbach, Komplementäre halbgruppen. kongruenzen and quotienten, Fundamenta Mathematicae, 69 (1970), 1-14.

[13] J. R. Büchi, T. M. Owens, Complemented monoids and hoops, unpublished manuscript, (1975).

[14] F. Feng, Y. B. Jun, X. Z. Zhao, Soft semirings, Computers and Mathematics with Applications, 56 (2008), 2621-2628.

[15] G. Georgescu, L. Leustean, V. Preoteasa, Pseudo-hoops, Journal of Multiple-Valued Logic and Soft Computing, 11(1-2) (2005), 153-184.

[16] A. R. Hadipour, A. Borumand Saeid, Fuzzy soft BF-algebra, Indian Journal of Science and Technology, 6(3) (2013), 4199-4204.

[17] P. Hájek, Metamathematics of fuzzy logic, Springer, 4 (1998).

[18] Y. B. Jun, Soft BCK/BCI-algebras, Computers and Mathematics with Applications, 56 (2008), 1408-1413.

[19] Y. B. Jun, K. J. Lee, C. H. Park, Fuzzy soft set theory applied to $B C K / B C I$-algebras, Computers and Mathematics with Applications, 59 (2010), 3180-3192.

[20] Y. B. Jun, C. H. Park, Applications of soft sets in ideal theory of BCK/BCI-algebras, Information Sciences, 178 (2008), 2466-2475.

[21] D. Molodtsov, Soft set theory - First results, Computers and Mathematics with Applications, 37 (1999), 19-31.

[22] D. Pei, D. Miaa, From soft sets to information systems, Proceedings of Granular Computing, 2 (2005), 617-621.

[23] A. Namdara, R. A. Borzooei, A. Borumand Saeid, M. Aaly Kologani, Some results in hoop algebras, Journal of Intelligent and Fuzzy Systems, 32 (2017), 1805-1813.

[24] S. Yamak, O. Kazanci, B. Davvaz, Soft hyper-structure, Computers and Mathematics with Applications, 62 (2011), 797-803.

[25] J. Zhan, W. A. Dudek, Soft MTL-Algebras based on fuzzy sets, Scientific Bulletin Series A, 74(2) (2012), 41-56. 
[26] J. Zhan, Y. B. Jun, Soft-algebras based on fuzzy sets, Computers and Mathematics with Applications, 59 (2010), 2037-2046.

R. A. Borzooei, E. Babaei, M. Mohseni Takallo

Department of Mathematics,

Shahid Beheshti University,

Tehran, Iran.

Emails:

borzooei@sbu.ac.ir

e_babaei@sbu.ac.ir

mohammad.mohseni1122@gmail.com

Y. B. Jun,

Department of Mathematics Education,

Gyeongsang National University,

Jinju 52828, Korea.

Email: skywine@gmail.com

M. Aaly Kologani

Hatef Higher Education Institute,

Zahedan, Iran.

Email: mona4011@gmail.com 